\title{
December 2014 Imaging Case of the Month
}

\author{
Michael B. Gotway, MD \\ Department of Radiology \\ Mayo Clinic Arizona \\ Scottsdale, AZ
}

Clinical History: A 34-year-old non-smoking woman presented to her physician as an outpatient with complaints of intermittent chest pain and intermittent mild hemoptysis. Her previous medical history was otherwise unremarkable.

Frontal chest radiography (Figure 1) was performed.

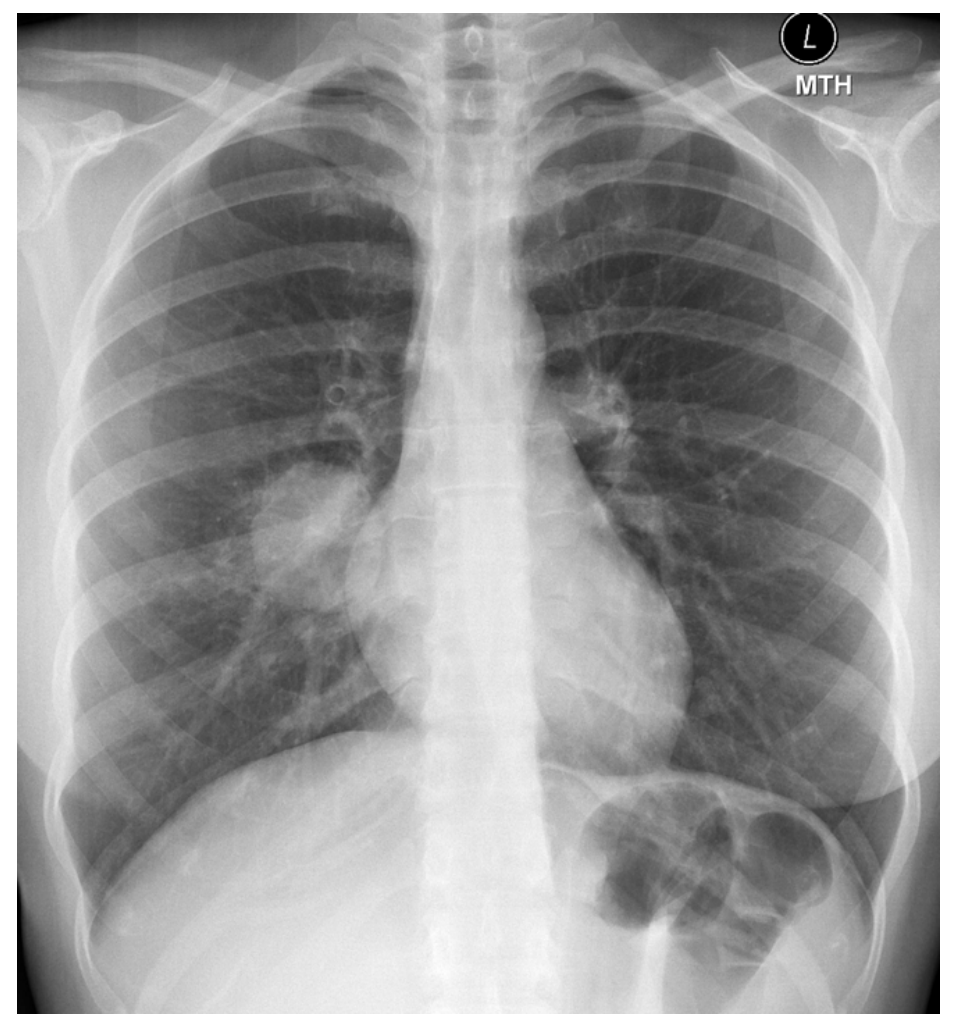

Figure 1. Frontal chest radiography.

Which of the following statements regarding the chest radiograph is most accurate?

1. The chest radiograph shows a circumscribed pulmonary mass

2. The chest radiograph shows asymmetric pulmonary vascularity

3. The chest radiograph shows bilateral linear and reticular opacities and diminished lung volumes suggesting fibrotic lung disease

4. The chest radiograph shows mild streaky central opacities, possibly reflecting airway thickening

5. The chest radiograph shows numerous small nodules 


\section{Correct! \\ 1. The chest radiograph shows a circumscribed pulmonary mass}

The chest radiograph shows a circumscribed soft tissue mass projected over the lateral aspect of the right pulmonary hilum. No evidence of streaky opacities to suggest bronchial wall thickening are evident, nor are small pulmonary nodules seen. The pulmonary vascularity appears normal and symmetric, and no features to suggest fibrotic lung disease are present.

Which of the following statements regarding the findings at chest radiography (Figure 1) is most accurate?

1. The chest radiograph shows the "cervico-thoracic" sign

2. The chest radiograph shows the "hilum overlay" sign

3. The chest radiograph shows the "incomplete border" sign

4. The chest radiograph shows the "Luftsichel" sign

5 . The chest radiograph shows the " $V$ sign of Naclerio" 


\section{Correct! \\ 2. The chest radiograph shows the "hilum overlay" sign}

The frontal chest radiograph shows the "hilum overlay" sign. Originally described as a means to distinguish a mediastinal mass (often anterior) from an enlarged heart and enlarged pulmonary arteries, this chest radiographic sign may be applied more generally and can be said to be present when a lesion detected at chest radiography resides anterior or posterior to the pulmonary hilum, but not within the hilum itself. When a lesion arises from the pulmonary hilar structures, the lesion will be in contact with the hilar pulmonary vessels, and will therefore exclude the air in the lung that normally surrounds these vessels and creates the tissue density difference that allows the pulmonary hilar vessels to be visualized at chest radiography. Therefore, a true hilar mass will obscure the margins of hilar vessels. When a lesion appears to be related to the hilum, but in fact the hilar vessel contours can clearly be seen "though" the lesion, then it follows that the lesion cannot arise from the hilum because lung air must still invest the hilar vessels- this situation represents the "hilum overlay" sign. The mass on the chest radiograph (Figure 1) shows this relationship- the right interlobar pulmonary artery can be clearly seen "thorough" the lung mass. The "incomplete border" sign is present when an opacity on chest radiography shows a relatively circumscribed border along one side, but the other side shows an indistinct border. This imaging finding may be seen with extraparenchymal lesions. The " $V$ sign of Naclerio" refers to $V$-shaped lucency projected over the basal medial left mediastinum, resulting from pneumomediastinum: one limb of the " $\mathrm{V}$ " is caused by air interposed between the parietal pleura and medial left diaphragm, whereas the other limb of the " $\mathrm{V}$ " results from air extending cranially along the medial left mediastinal border. The "Luftsichel" sign may be seen in patients with left upper lobe collapse. Luftsichel in German means "air crescent," ("luft" = air, "sichel"= sickle) which refers to the lucency that may outline the aortic arch when the superior segment of the left lower lobe becomes hyperinflated and extends more cranially than usual in response to left upper lobe collapse. The "cervicothoracic sign describes the location of a lesion at the thoracic inlet. Because the posterior lung apex normally extends more cranially than the anterior lung apex, a lesion in the posterior mediastinum located at the cervico-thoracic junction will show a contour abnormality that extends more superiorly than an anterior or middle mediastinal lesion. This occurs because a posterior mediastinal lesion in this location will maintain contact with the lung parenchyma to a greater superior extent [generally cranial to the clavicles] than either anterior or middle mediastinal lesions at the cervico-thoracic junction, thereby creating a visible contour, whereas anterior or middle mediastinal lesions at the cervico-thoracic junction will lose their visible contour as they enter the soft tissues of the caudal neck.

Clinical course: The patient subsequently underwent unenhanced thoracic CT (Figure 2). 


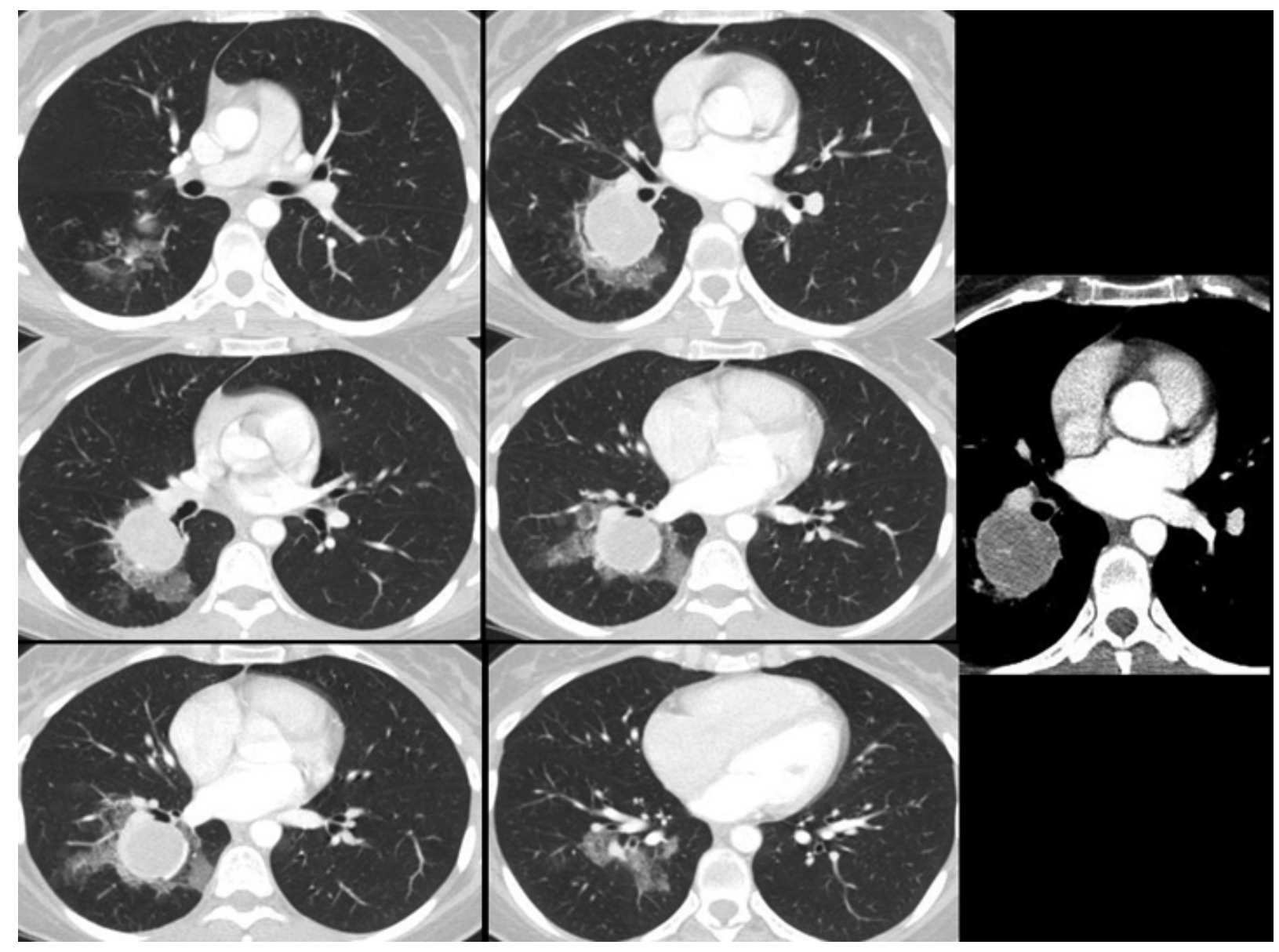

Figure 2: Axial contrast-enhanced thoracic CT.

Which of the following is the leading consideration for the likely etiology of the lesion in this patient?

1. Bronchogenic carcinoma

2. Carcinoid tumor

3. Chronic necrotizing aspergillosis

4. Inflammatory myofibroblastic tumor

5. Solitary fibrous tumor 


\section{Correct! \\ 2. Carcinoid tumor}

Among the choices listed, carcinoid tumor is the most likely diagnosis. The close association of the mass with the superior segmental right lower lobe bronchus (Figure 3, arrow) suggests a lesion originating from the airway, and the patchy areas of groundglass opacity that surround the mass (Figure 3 , arrowheads) raises the possibility of hemorrhage associated with the lesion, which could reflect the vascular nature of a carcinoid tumor.

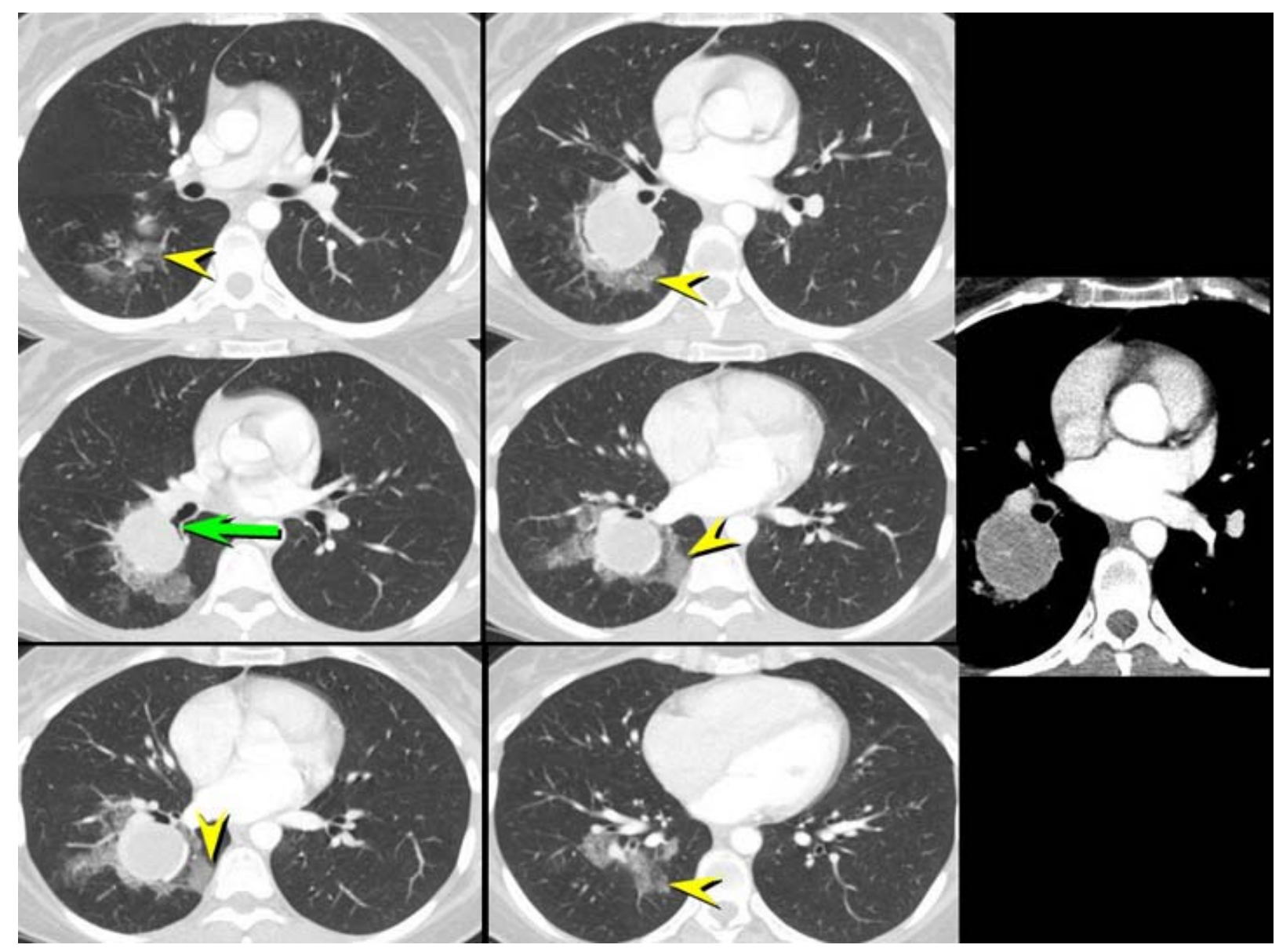

Figure 3. Axial contrast-enhanced thoracic CT shows a circumscribed superior segmental right lower lobe mass closely associated with the superior segmental right lower lobe bronchus (arrow). Patchy areas of ground-glass opacity (arrowheads) are

present along the margins of the lesion, suggesting peri-lesional hemorrhage. The lesion shows no calcification and is relatively homogeneous-appearing on the soft tissue window image.

Furthermore, the patient's relatively young age is appropriate for carcinoid tumor, but is atypically young for bronchogenic carcinoma. Inflammatory myofibroblastic tumor is a possibility, but the imaging features of this disorder are relatively non-specific and this lesion is considerably rarer than carcinoid tumor. Furthermore, inflammatory 
myofibroblastic tumor is frequently associated with calcification, which is not seen within this lesion. Chronic necrotizing aspergillosis typically occurs in middle-aged to older patients with relatively mild degrees of immunosuppression, such as chronic steroid use, alcoholism, chronic liver disease, diabetes mellitus, etc., or underlying structural lung disease, such as obstructive pulmonary disease, previous infection, prior radiation therapy, chronic inflammatory conditions (e.g., sarcoidosis), and occupational lung diseases; such history is not present in this case. Additionally, chronic necrotizing aspergillosis often appears as a slowly growing nodular opacity that subsequently cavitates by the time it reaches a substantial size, but cavitation is not seen in this lesion. Solitary fibrous tumors may arise in a variety of organ systems, but are most commonly encountered arising from the pleura, and therefore appear as an extraparenchymal mass, unlike the lesion in this case.).

Which of the following represents the next most appropriate step for the evaluation of this patient?

1. ${ }^{18}$ FDG-PET scanning

2. ${ }^{68} \mathrm{Ga}$ scanning

3. Repeat chest radiography

4. Repeat CT using a solitary pulmonary nodule enhancement protocol

5. Thoracic MRI 


\section{Correct! \\ 1. ${ }^{18}$ FDG-PET scanning}

${ }^{18} \mathrm{FDG}-\mathrm{PET}$ scanning is the most appropriate procedure among those listed above for the evaluation of this patient. ${ }^{18}$ FDG-PET scanning has the ability to assess for additional sites of hypermetabolic tissue, perhaps outside of the thorax and potentially unsuspected, that may change the differential diagnostic considerations or provide an additional target for tissue sampling. The presence of elevated tracer utilization within the lesion may favor neoplasia and prompt a more aggressive approach to obtaining a diagnosis, although the lack for significant tracer utilization within this lesion would not obviate the need for further evaluation. Repeat chest radiography is often a useful tactic when an opacity seen at chest radiography is thought to be artifactual. Artifacts, such as superimposition, generally do not persist on repeat imaging, and therefore this simple method allows patient disposition without use of more expensive procedures associated with higher radiation exposures. Thoracic MRI may be used for a number of thoracic disorders, such as mediastinal mass assessment, lung cancer staging (particularly for potential chest wall or mediastinal invasion), and a number of other disorders, but is generally utilized for solitary pulmonary nodule or mass evaluation. Furthermore, it is unlikely that thoracic MRI will add significant information to what is already available with the contrast-enhanced thoracic CT. Repeating the thoracic CT using a nodule enhancement protocol, which typically involves the initial use of unenhanced imaging followed by a contrast-enhanced study using a specific weight-based contrast injection technique within imaging at 1 minute intervals for 4 minutes- the intent is to assess for significant enhancement within the lesion (usually defined as more than $15 \mathrm{HU}$ of enhancement within the lesion at any of the 4 time points). The lack of significant enhancement is strongly suggestive of a benign etiology for a lung lesion; however, enhancement within a focal lung opacity is relatively non-specific and can occur with both benign and malignant lung lesions. Typically this protocol is used for smaller indeterminate focal lung opacities, where negative results will allow conservative management, such as serial observation. In this patient, given the size of the lesion and the presence of symptoms, a conservative management approach consisting of serial observation for growth would not be appropriate. Finally, because CT nodule enhancement protocols are associated with repeatedly scanning the chest, they are potentially associated with significant patient radiation exposures, even when performed using low-dose techniques; therefore, the use of this protocol should be restricted to situations where the potential cost-to-benefit ratio is clear. ${ }^{68} \mathrm{Ga}$-citrate scanning (aka "gallium" scan) could show uptake in the right lower lobe lesion, but such uptake is relatively non-specific, and may be seen with inflammatory or malignant etiologies, and negative results would not provide any useful data. The most common use of ${ }^{68} \mathrm{Ga}$ citrate scanning for thoracic disorders usually occurs in the context of diffuse lung disease, not focal pulmonary disorders.).

Further clinical course: The patient subsequently underwent ${ }^{18}$ FDG-PET scanning which showed relatively low-level tracer uptake within the right lower lobe lesion, which was interpreted as not suggestive of malignancy. 
Which of the following represents the next most appropriate step for the evaluation of this patient?

1. Bronchoscopy with transbronchial biopsy

2. Percutaneous transthoracic needle biopsy

3. Thoracotomy

4. $a$ or $b$

5. b or c 


\section{Correct! \\ 4. $a$ or $b$}

Because the superior segmental right lower lobe lesion is relatively central in location and clearly associated with an airway, bronchoscopy with transbronchial biopsy would be an appropriate technique to employ to obtain a tissue diagnosis. Similarly, the lesion is readily approachable using transthoracic fine needle biopsy, using both aspiration and core biopsy techniques. While thoracotomy certain could obtain a definitive diagnosis for this patient, such an approach is needlessly invasive and expensive and should be employed when other less invasive approaches have failed to obtain a definitive diagnosis.).

Further clinical course. The patient underwent bronchoscopy with transbronchial biopsy, but material sufficient for confident diagnosis could not be obtained. Subsequently, the patient then underwent transthoracic fine needle biopsy using both aspiration and core biopsy techniques, which yielded tissue showing two epithelial cell populations: one consisting of pale round interstitial cells of a respiratory epithelial phenotype, and another consisting of surface cuboidal cells derived from Clara cells. These cell populations were embedded in a sclerotic stroma. The histologic features were considered diagnostic of pulmonary sclerosing hemangioma.

Diagnosis: Pulmonary sclerosing hemangioma

\section{References}

1. Algın O, Gökalp G, Topal U. Signs in chest imaging. Diagn Interv Radiol. 2011; 17(1):18-29. [PubMed]

2. Hsu CC, Henry TS, Chung JH, Little BP. The incomplete border sign. J Thorac Imag. 2014; 29(4):W48. [CrossRef] [PubMed]

3. Parker MS, Chasen MH, Paul N. Radiologic signs in thoracic imaging: case-based review and self-assessment module. AJR Am J Roentgenol. 2009; 192(3Suppl):S3448. [CrossRef] [PubMed]

4. Kousha M, Tadi R, Soubani AO. Pulmonary aspergillosis: a clinical review. Eur Respir Rev. 2011; 20(121):156-74. [CrossRef] [PubMed]

5. Swensen SJ, Viggiano RW, Midthun DE, Müller NL, Sherrick A, Yamashita K,Naidich DP, Patz EF, Hartman TE, Muhm JR, Weaver AL. Lung nodule enhancement at CT: multicenter study. Radiology. 2000; 214(1):73-80. [CrossRef] [PubMed]

6. Chen B, Gao J, Chen H, Cao Y, He X, Zhang W, Luo M, Zhang S, Li W. Pulmonary sclerosing hemangioma: a unique epithelial neoplasm of the lung (report of 26 cases). World J Surg Oncol. 2013; 11:85-92. [CrossRef] [PubMed] 